\title{
Enhancing The Predictability of CRUde OIL MARKETS WITH HYBRID WAVELET APPROACHES
}

... In honor of Professor Ramo Gençay

\author{
GAZI SALAH UDDIN $^{\mathrm{a}}{ }^{*}$ RAMAZAN GENÇAY $^{\mathrm{b}}$ R $^{\dagger}$ \\ STELIOS BEKIROS $^{\mathrm{c}, \mathrm{d} \dot{\dagger}}$ MAZIAR SAHAMKHADAM $^{\mathrm{e} \S}$ \\ ${ }^{a}$ Linköping University, Sweden \\ ${ }^{b}$ Simon Fraser University, Canada \\ ${ }^{c}$ European University Institute, Italy \\ ${ }^{d}$ Athens University of Economics \& Business, Greece \\ ${ }^{e}$ Linnaeus University, Sweden
}

\begin{abstract}
We explore the robustness, efficiency and accuracy of the multi-scale forecasting in crude oil markets. We adopt a novel hybrid wavelet auto-ARMA model to detect the inherent nonlinear dynamics of crude oil returns with an explicitly defined hierarchical structure. Entropic estimation is employed to calculate the optimal level of the decomposition. The wavelet-based forecasting method accounts for the chaotic behavior of oil series, whilst captures drifts, spikes and other non-stationary effects which common frequency-domain methods miss out completely. These results shed new light upon the predictability of crude oil markets in nonstationary settings.
\end{abstract}

JEL Classification: C53; G17

Keywords: Wavelet decomposition; Forecasting; Crude oil

\footnotetext{
* This work is dedicated to the loving memory of Ramo Gençay whose everlasting contribution to multiscale resolution analysis in economics inspired not only His collaborating co-authors in this paper, but academics worldwide over the last decades.

Gazi Salah Uddin is thankful for the financial support provided by the Jan Wallander and Tom Hedelius Foundations and Siamon Foundation. The usual disclaimer applies.

a Corresponding author: Department of Management and Engineering, Linköping University, Sweden; gazi.salah.uddin@liu.se

$\uparrow^{b}$ Department of Economics, Simon Fraser University, Canada; rgencay@sfu.ca

$\${ }^{c}$ Department of Economics, EUI, Florence, Italy; ${ }^{d}$ School of Business Administration, AUEB, Athens, Greece; stelios.bekiros@eui.eu

${ }^{\S}$ Department of Accounting and Logistics, Linnaeus University, Sweden; maziar.sahamkhadam@lnu.se
} 


\title{
Enhancing The Predictability of Crude OIL MARKETS WITH HYBRID WAVELET APPROACHES
}

... In honor of Professor Ramo Gençay

\author{
GAZI SALAH UDDIN $^{\mathrm{a}}{ }^{*}$ RAMAZAN GENÇAY ${ }^{\mathrm{b}}$ R $^{\dagger}$ \\ STELIOS BEKIROS $^{\mathrm{c}, \mathrm{d} \dot{\dagger}}$ MAZIAR SAHAMKHADAM $^{\mathrm{e} \S}$ \\ ${ }^{a}$ Linköping University, Sweden \\ ${ }^{b}$ Simon Fraser University, Canada \\ ${ }^{c}$ European University Institute, Italy \\ ${ }^{d}$ Athens University of Economics \& Business, Greece \\ ${ }^{e}$ Linnaeus University, Sweden
}

\begin{abstract}
We explore the robustness, efficiency and accuracy of the multi-scale forecasting in crude oil markets. We adopt a novel hybrid wavelet auto-ARMA model to detect the inherent nonlinear dynamics of crude oil returns with an explicitly defined hierarchical structure. Entropic estimation is employed to calculate the optimal level of the decomposition. The wavelet-based forecasting method accounts for the chaotic behavior of oil series, whilst captures drifts, spikes and other non-stationary effects which common frequency-domain methods miss out completely. These results shed new light upon the predictability of crude oil markets in nonstationary settings.
\end{abstract}

JEL Classification: C53; G17

Keywords: Wavelet decomposition; Forecasting; Crude oil

\footnotetext{
* This work is dedicated to the loving memory of Ramo Gençay whose everlasting contribution to multiscale resolution analysis in economics inspired not only His collaborating co-authors in this paper, but academics worldwide over the last decades.

Gazi Salah Uddin is thankful for the financial support provided by the Jan Wallander and Tom Hedelius Foundations and Siamon Foundation. The usual disclaimer applies.

a Corresponding author: Department of Management and Engineering, Linköping University, Sweden; gazi.salah.uddin@liu.se

$\uparrow^{b}$ Department of Economics, Simon Fraser University, Canada; rgencay@sfu.ca

$\${ }^{c}$ Department of Economics, EUI, Florence, Italy; ${ }^{d}$ School of Business Administration, AUEB, Athens, Greece; stelios.bekiros@eui.eu

${ }^{\S}$ Department of Accounting and Logistics, Linnaeus University, Sweden; maziar.sahamkhadam@lnu.se
} 


\section{INTRODUCTION}

The past decade witnessed a fundamental change in the role of energy markets, which were increasingly utilized by market agents for portfolio risk diversification purposes. In the wake of the Global Financial Crisis and the subsequent geopolitical tension, predicting crude oil prices rendered invaluable for policymakers and investors, as the business cycle of oil markets illustrates an interesting heterogeneity among other commodity classes.

Consequently, predicting oil markets is of paramount importance. The previous literature on forecasting methods for commodities prices suffers from various problems vis-à-vis the driving factors of financial markets. For instance, one recent study by Zhang et al. (2017) based on Hyndmann et al. (2011), criticizes existing approaches and suggests a way to decompose univariate time series into "a set of constitutive series" hence performing efficient forecasting. The novel approach could be utilized not only for stock indexes as in Zhang et al. (2017), but importantly for crude oil. However, it is important to properly assess and estimate the level of decomposition into various modes or components of the time series in question.

In our work, we introduce for the first time a new class of optimal multi-scale multiresolution forecasting approach designed to detect the inherent nonlinear dynamics of crude oil returns comprising a set of constitutive series with an explicitly defined hierarchical structure. We adopt a wavelet-based technique to produce forecast horizons via monthly information. We calculate the optimal level of the wavelet decomposition via entropic estimation and wavelet coherence. The rationale behind selecting a wavelet forecasting method lays in our intention to account for the chaotic behavior of oil series and capture drifts, spikes and other nonstationary effects that common frequency-domain decomposition methods are unable to reveal or miss out completely. The multi-scale multiresolution forecasting is related to several approaches that combine data at different frequencies, including the Mixed Data Sampling Regression Models (Ghysels et al., 2004), the Heterogeneous Autoregressive model of Realized Volatility (Corsi, 2009), the Mixed-frequency forecasting framework (Schorfheide \& Song, 2015), and the Bayesian multi-scale dynamics (Bianchi \& Tamoni, 2016). The application of multi-scale decomposition enhances predictability in any step-ahead horizon for crude oil markets.

The rest of the paper is organized as follows. Section 2 presents the novel methodology. The data and empirical results are described in section 3 , while section 4 concludes. 


\section{MeTHOdology}

Following Zhang et al. (2017) and Hyndmann et al. (2011), we combine multiresolution analysis (MRA) with ordinary least squares (OLS) regression modeling. The multiresolution analysis is used to decompose the original time-series into "details" and "smooths". By applying a univariate forecasting procedure, we obtain "first-round step-ahead forecasts" of the original and of the decomposed series. Zang et al. (2017) suggested that each variable at different scale can be considered as a linear combination of its lowest-scale counterparts. However, the hierarchical structure of the original time-series is not preserved in first-round forecasts in case of auto-regressive moving average (ARMA) models. To overcome this problem and obtain the optimal forecasts at all hierarchical levels, Zang et al. (2017) regressed the first-round forecasts of the series on a "summing" matrix, which depicts the underlying linear relationship in hierarchical structure. However, they only examined their approach up to 2-level wavelet decomposition. In this paper, we extend the method to higherlevel wavelet-based multiresolution analysis.

\subsection{MRA-Wavelet Decomposition}

By using MRA for the training sample $X_{t}, t \in[1, T]$, we obtain the wavelet details, $D_{j, t}$, and smooths, $S_{j, t}$. We use a maximal overlap discrete wavelet transform (MODWT) to obtain $j$ th level MODWT wavelet $W_{j, t}$ and scaling $V_{j}$ coefficients (Durai \& Bhaduri, 2009) as:

$$
\begin{gathered}
W_{j, t}=\sum_{l=0}^{L_{1}-l} \tilde{k}_{j, l} X_{t-l \bmod N} \quad V_{j, t}=\sum_{l=0}^{L_{1}-l} \tilde{g}_{j, l} X_{t-l \bmod N} \\
D_{j, t}=\sum_{l=0}^{N-l} \tilde{k}_{j, l} W_{j, t+l \bmod N}=\sum_{l=0}^{N-l} \tilde{g}_{j, l} V_{j, t+l \bmod N} \\
X_{t}=\sum_{j=1}^{J} D_{j, t}+S_{j, t}
\end{gathered}
$$


where, $\tilde{k}_{j, l}=k_{j, l} / 2^{j / 2}, \tilde{g}_{j, l}=g_{j, l} / 2^{j / 2}$ denote the wavelet and scaling filters, respectively, $j \in[2, J]$ and $J$ is the level of decomposition.

\subsection{Auto-ARMA}

Having obtain the MRA-Wavelet based decomposed series, $D_{j, t}$, and $S_{j, t}$, we use the estimation of auto-ARMA by Khandakar and Hyndman (2008), whereby the optimal AR and MA orders are selected based on the Akaike's information criterion (AIC). We apply the autoARMA model both to the original, $X_{t}$, and the decomposed series:

$$
\begin{gathered}
D_{j, t}=c+\sum_{i=1}^{p} \varphi_{i} D_{j, t-i}+\sum_{i=1}^{q} \theta_{i} \varepsilon_{t-i}+\varepsilon_{t} \\
S_{j, t}=c+\sum_{i=1}^{p} \varphi_{i} S_{j, t-i}+\sum_{i=1}^{q} \theta_{i} \varepsilon_{t-i}+\varepsilon_{t} \\
X_{t}=c+\sum_{i=1}^{p} \varphi_{i} X_{t-i}+\sum_{i=1}^{q} \theta_{i} \varepsilon_{t-i}+\varepsilon_{t}
\end{gathered}
$$

where, $t \in[1, T]$. By applying the auto-ARMA forecasting modeling in Eqs. 4-6, we obtain “ “first-round forecasts", namely $\widehat{Y}_{T+h}=\left[\widehat{X}_{T+h}, \widehat{D}_{1, T+h}, \ldots, \widehat{D}_{J, T+h}, \hat{S}_{1, T+h}, \ldots, \hat{S}_{J, T+h}\right]^{\prime}$.

\subsection{OLS}

Based on the algorithm presented by Zang et al. (2017), we construct the "summing" matrix $Z$, with 0 and 1 entries, which captures the linear relationship under a hierarchical structural scheme. Considering the base-level variables, $\beta_{t}=\left[S_{J, t}, D_{J, t}, D_{J-1, t}, \ldots, D_{1, t}\right]^{\prime}$, the linear relationship can be expressed as: 
$Y_{t} \equiv\left[\begin{array}{c}X_{t} \\ S_{1, t} \\ D_{1, t} \\ \vdots \\ S_{J, t} \\ D_{J, t}\end{array}\right]=\left[\begin{array}{c}S_{J, t}+D_{J, t}+D_{J-1, t}+\cdots+D_{1, t} \\ S_{J, t}+D_{J, t}+D_{J-1, t}+\cdots+D_{2, t} \\ D_{1, t} \\ \vdots \\ S_{J, t} \\ D_{J, t}\end{array}\right]=\left[\begin{array}{ccccc}1 & 1 & 1 & \ldots & 1 \\ 1 & 1 & 1 & \ldots & 0 \\ 0 & 0 & 0 & \ldots & 1 \\ \vdots & \vdots & \vdots & \ddots & \vdots \\ 1 & 0 & 0 & \cdots & 0 \\ 0 & 1 & 0 & \ldots & 0\end{array}\right]\left[\begin{array}{c}S_{J, t} \\ D_{J, t} \\ D_{J-1, t} \\ \vdots \\ D_{1, t}\end{array}\right] \equiv Z \beta_{t}$

By using OLS, we regress the first-round forecasts, $\widehat{Y}_{T+h}=\left[\widehat{X}_{T+h}, \widehat{D}_{1, T+h}, \ldots, \widehat{D}_{J, T+h}, \hat{S}_{1, T+h}, \ldots, \hat{S}_{J, T+h}\right]^{\prime}$ upon the summing matrix $Z$. This provides us with optimal base-level forecasts, $\widetilde{\beta}_{T+h}=\left[\tilde{S}_{J, T+h}, \widetilde{D}_{J, T+h}, \widetilde{D}_{J-1, T+h}, \ldots, \widetilde{D}_{1, T+h}\right]^{\prime}$. Next by utilizing the best base-level forecasts from the OLS, we estimate the optimal forecasts at all hierarchical levels as follows:

$$
\tilde{Y}_{T+h}=Z \widetilde{\beta}_{T+h}=\left[\begin{array}{ccccc}
1 & 1 & 1 & \ldots & 1 \\
1 & 1 & 1 & \ldots & 0 \\
0 & 0 & 0 & \ldots & 1 \\
\vdots & \vdots & \vdots & \ddots & \vdots \\
1 & 0 & 0 & \ldots & 0 \\
0 & 1 & 0 & \ldots & 0
\end{array}\right]\left[\begin{array}{c}
\tilde{S}_{J, T+h} \\
\widetilde{D}_{J, T+h} \\
\widetilde{D}_{J-1, T+h} \\
\vdots \\
\widetilde{D}_{1, T+h}
\end{array}\right]=\left[\begin{array}{c}
\widehat{X}_{T+h} \\
\tilde{S}_{1, T+h} \\
\widetilde{D}_{1, T+h} \\
\vdots \\
\tilde{S}_{J, T+h} \\
\widetilde{D}_{J, T+h}
\end{array}\right]
$$

\section{EMPIRICAL RESULTS}

Our dataset comprises monthly WTI prices of Crude Oil (ODA/POILWTI_USD) obtained from QUANDL data base ${ }^{1}$. The monthly series span February 29, 1980 to June 30, 2017. We use log returns to compute our investigated series. We apply the Shannon entropy criterion as in Bekiros and Marcellino (2013) and the wavelet continuous coherence under uncertainty (Bekiros et al., 2015) to estimate the optimal level of decomposition for the specific data frequency of the oil price returns. We render the optimal decomposition level as eight ${ }^{2}$. From the optimal forecasts obtained in Eq. 8, we generate the top-level forecasts as $\hat{X}_{T+h}=$ $\tilde{S}_{J, T+h}+\widetilde{D}_{J, T+h}+\cdots, \widetilde{D}_{1, T+h}$, that can be compared directly to the original time-series over the out-of-sample period. As we are interested in testing the out-of-sample performance of the suggested forecasting approach at different wavelet-decomposition levels $(J=2,3, \ldots, 8)$ we apply a rolling window estimation and perform an $h$-step ahead $(h=1,2, \ldots, 14)$ forecasting

\footnotetext{
${ }^{1}$ We have also tested the daily crude oil prices (DOE/RWTC) obtained from QUANDL database. Based on daily oil prices, in almost all cases the simple auto-ARMA modeling outperforms the auto ARMA-MRA model with respect to reducing forecast errors.

${ }^{2}$ The detailed results are available upon request.
} 
scheme at each out-of-sample iteration. As in similar works mentioned above, the training sample size is dyadic in this application, i.e., $T=2^{J}, J=2,3, \ldots, 8$. For the out-of-sample performance of the top-level forecasts, we consider the mean absolute error (MAE), root mean squared error (rMSE) and compare them against those obtained from the plain vanilla auto-ARMA model. ${ }^{3}$

Tables 1-2 provide forecast enhancement results. It is evident from our analysis that the parameter estimates as depicted in the tables are either higher (improvement) or lower (i.e., no improvement) 1 . As it is shown using 2, 3 and 4 levels of decomposition, in all cases the auto ARMA-MRA model provides with better forecasts compared to the simple ARMA model. Tables 3-4 report the statistical significance of the improvement obtained from the hybrid auto ARMA-MRA with different decomposition levels relatively to the benchmark.

In figures 1-2, forecast errors (rMSE and MAE) are given for 3 and 4 levels of decomposition of the monthly crude oil prices. Clearly, we see the enhancement obtained by applying the auto ARMA-MRA hybrid. Figure 3 displays the auto ARMA-MRA model with 2 levels of decomposition and several window lengths.

\section{Conclusions}

We introduce a hybrid wavelet-based MRA to forecast monthly crude oil prices. Our results suggest that the auto ARMA-MRA model is more accurate than the simple one, in terms of forecastability. The predictive accuracy would decrease leading to higher forecast errors by increasing the level of decomposition, which could be due to the requirement of increasing the estimation window length. As the market participants entail diverse trading objectives (Aguiar-Conraria and Soares, 2013; Bekiros et al., 2016), our findings provide interesting insights especially for long-term investors who focus on low-frequency (high-scale) long-run oscillations. These results may have important implications for the market predictability of crude oil markets.

\footnotetext{
${ }^{3}$ To evaluate the economic performance of the suggested models, one can estimate the out-of-sample Sharpe ratios (see Gu et al., 2018; Bianchi et al., 2018). This Sharpe-based $R^{2}$ in some cases generated extremely close to zero scores (i.e., slightly positive or slightly negative values), hence proper estimation of comparative Sharpebased $R^{2}$ was not achieved in all instances. The economic performance of other forecasting models vis-à-vis the hybrid wavelet-based MRA, is considered in future research.
} 


\section{REFERENCES}

Aguiar-Conraria, L., and Soares, M. J., 2014. The continuous wavelet transform: moving beyond uni and bivariate analysis. Journal of Economic Surveys, 28(2), 344-375.

Bekiros, S., Marcellino, M., 2013. The multiscale causal dynamics of foreign exchange markets. Journal of International Money and Finance, 33, 282-305.

Bekiros, S., Nguyen, D. K., Uddin, G. S., Sjö, B., 2015. Business cycle (de) synchronization in the aftermath of the global financial crisis: implications for the Euro area. Studies in Nonlinear Dynamics \& Econometrics, 19(5), 609-624.

Bekiros, S., Nguyen, D. K., Uddin, G. S., Sjö, B., 2016. On the time scale behavior of equitycommodity links: Implications for portfolio management. Journal of International Financial Markets, Institutions and Money, 41, 30-46.

Bianchi, D. and Tamoni, A., 2016. The dynamics of expected returns: Evidence from multiscale time series modeling. Available at SSRN 2684728.

Bianchi, D., Büchner, M., \& Tamoni, A., 2018. Bond Risk Premia with Machine Learning. Available at SSRN 3232721.

Corsi, F., 2009. A simple approximate long-memory model of realized volatility. Journal of Financial Econometrics, 7(2), pp.174-196.

Durai, S. R. S., Bhaduri, S. N., 2009. Stock prices, inflation and output: Evidence from wavelet analysis. Economic Modelling, 26(5), 1089-1092.

Gençay, R., Selçuk, F., Whitcher, B. J., 2001. An Introduction to Wavelets and Other Filtering Methods in Finance and Economics. Academic press.

Ghysels, E., Santa-Clara, P. and Valkanov, R., 2004. The MIDAS touch: Mixed data sampling regression models.

Gu, S., Kelly, B., \& Xiu, D., 2018. Empirical asset pricing via machine learning. National Bureau of Economic Research, No. w25398.

Hyndman, R. J., Ahmed, R. A., Athanasopoulos, G., Shang, H. L., 2011. Optimal combination forecasts for hierarchical time series. Computational Statistics \& Data Analysis, 55(9), 2579-2589.

Khandakar, Y., Hyndman, R. J., 2008. Automatic time series forecasting: the forecast Package for R. Journal of Statistical Software, 27(03),1-22.

Schorfheide, F. and Song, D., 2015. Real-time forecasting with a mixed-frequency VAR. Journal of Business \& Economic Statistics, 33(3), pp.366-380.

Zhang, K., Gençay, R., Yazgan, M. E., 2017. Application of wavelet decomposition in timeseries forecasting. Economics Letters, 158, 41-46. 


\section{TABLE 1. FORECAST IMPROVEMENT (RMSE) FOR CRUDE OIL MARKETS}

\begin{tabular}{|c|c|c|c|c|c|c|c|c|c|c|c|c|c|c|c|c|}
\hline \multirow{2}{*}{ Forecasting Model } & \multirow{2}{*}{$\begin{array}{l}\text { Window } \\
\text { Length }\end{array}$} & \multicolumn{14}{|c|}{ Forecast Horizon } & \multirow{2}{*}{-Average } \\
\hline & & 1 & 2 & 3 & 4 & 5 & 6 & 7 & 8 & 9 & 10 & 11 & 12 & 13 & 14 & \\
\hline auto ARMA-MRA (8) & \multirow{2}{*}{256} & 0.939 & 1.004 & 0.976 & 0.961 & 0.969 & 0.960 & 0.976 & 0.990 & 0.999 & 0.996 & 0.994 & 0.991 & 0.988 & 0.990 & 0.981 \\
\hline auto ARMA-MRA (2) & & 0.968 & 1.011 & 0.999 & 0.987 & 0.986 & 0.986 & 0.993 & 1.003 & 1.005 & 1.004 & 1.001 & 0.999 & 0.992 & 0.994 & 0.995 \\
\hline auto ARMA-MRA (7) & \multirow{2}{*}{128} & 0.922 & 1.001 & 1.006 & 1.005 & 1.008 & 1.005 & 0.998 & 1.002 & 1.001 & 1.002 & 1.005 & 1.010 & 1.003 & 1.004 & 0.998 \\
\hline auto ARMA-MRA (2) & & 0.973 & 1.009 & 1.004 & 1.004 & 1.005 & 1.001 & 0.997 & 1.004 & 1.005 & 1.004 & 1.006 & 1.007 & 1.001 & 1.002 & 1.002 \\
\hline auto ARMA-MRA (6) & \multirow{2}{*}{64} & 0.942 & 1.036 & 1.043 & 1.009 & 1.003 & 0.997 & 0.990 & 1.016 & 1.014 & 1.021 & 1.018 & 1.002 & 1.007 & 1.014 & 1.007 \\
\hline auto ARMA-MRA (2) & & 0.981 & 1.030 & 1.034 & 1.007 & 1.006 & 1.000 & 0.989 & 1.006 & 1.007 & 1.008 & 1.008 & 0.996 & 0.998 & 0.999 & 1.005 \\
\hline auto ARMA-MRA (5) & \multirow{2}{*}{32} & 0.973 & 1.019 & 1.060 & 1.039 & 1.033 & 1.007 & 1.007 & 1.011 & 1.019 & 1.014 & 1.011 & 0.992 & 1.005 & 1.027 & 1.015 \\
\hline auto ARMA-MRA (2) & & 0.997 & 1.018 & 1.051 & 1.034 & 1.023 & 1.002 & 1.003 & 1.007 & 1.010 & 1.005 & 1.007 & 0.995 & 1.008 & 1.019 & 1.013 \\
\hline auto ARMA-MRA (4) & \multirow{2}{*}{16} & 1.001 & 1.042 & 1.079 & 1.073 & 1.126 & 1.165 & 1.195 & 1.223 & 1.229 & 1.281 & 1.291 & 1.277 & 1.289 & 1.310 & 1.192 \\
\hline auto ARMA-MRA (2) & & 1.012 & 1.036 & 1.067 & 1.060 & 1.105 & 1.127 & 1.156 & 1.176 & 1.182 & 1.215 & 1.227 & 1.228 & 1.237 & 1.258 & 1.156 \\
\hline auto ARMA-MRA (3) & \multirow{2}{*}{8} & 1.028 & 1.064 & 1.108 & 1.133 & 1.160 & 1.231 & 1.266 & 1.303 & 1.340 & 1.382 & 1.406 & 1.441 & 1.466 & 1.502 & 1.289 \\
\hline auto ARMA-MRA (2) & & 1.025 & 1.057 & 1.094 & 1.116 & 1.139 & 1.195 & 1.226 & 1.256 & 1.284 & 1.318 & 1.334 & 1.363 & 1.381 & 1.408 & 1.243 \\
\hline
\end{tabular}

Notes: This table reports the improvements in rMSE based on the auto ARMA-MRA model vs. simple ARMA. The numbers presented are the ratio of rMSE based on the simple ARMA model over the rMSE of ARMA-MRA model. We use the MODWT with haar wavelet. In the parentheses, we show the levels of the decomposition. Window length and forecast horizon are in monthly basis. The last column reports the average improvement in rMSE. 


\section{TABLE 2. ForeCAST IMPROVEMENT (MAE) FOR CRUDE OIL MARKETS}

\begin{tabular}{|c|c|c|c|c|c|c|c|c|c|c|c|c|c|c|c|c|}
\hline \multirow{2}{*}{ Forecasting Model } & \multirow{2}{*}{$\begin{array}{l}\text { Window } \\
\text { Length }\end{array}$} & \multicolumn{14}{|c|}{ Forecast Horizon } & \multirow{2}{*}{-Average } \\
\hline & & 1 & 2 & 3 & 4 & 5 & 6 & 7 & 8 & 9 & 10 & 11 & 12 & 13 & 14 & \\
\hline auto ARMA-MRA (8) & \multirow{2}{*}{256} & 0.934 & 0.973 & 0.963 & 0.961 & 0.972 & 0.962 & 0.984 & 0.992 & 1.009 & 0.999 & 1.002 & 0.993 & 0.989 & 0.989 & 0.980 \\
\hline auto ARMA-MRA (2) & & 0.962 & 0.992 & 0.992 & 0.987 & 0.989 & 0.983 & 0.993 & 1.001 & 1.011 & 1.008 & 1.007 & 1.000 & 0.995 & 0.992 & 0.994 \\
\hline auto ARMA-MRA (7) & \multirow{2}{*}{128} & 0.921 & 0.989 & 0.997 & 1.001 & 1.002 & 1.007 & 0.996 & 1.008 & 1.005 & 0.999 & 1.004 & 1.007 & 1.000 & 1.004 & 0.995 \\
\hline auto ARMA-MRA (2) & & 0.964 & 1.000 & 1.006 & 1.008 & 1.007 & 1.005 & 0.998 & 1.008 & 1.006 & 1.002 & 1.006 & 1.005 & 0.999 & 1.004 & 1.001 \\
\hline auto ARMA-MRA (6) & \multirow{2}{*}{64} & 0.944 & 1.007 & 1.017 & 0.997 & 0.995 & 0.995 & 0.980 & 1.016 & 1.013 & 1.029 & 1.026 & 1.003 & 1.005 & 1.012 & 1.002 \\
\hline auto ARMA-MRA (2) & & 0.979 & 1.002 & 1.016 & 1.006 & 1.004 & 1.002 & 0.983 & 1.005 & 1.007 & 1.010 & 1.017 & 0.997 & 1.000 & 0.998 & 1.002 \\
\hline auto ARMA-MRA (5) & \multirow{2}{*}{32} & 0.963 & 0.998 & 1.034 & 1.036 & 1.014 & 0.995 & 1.002 & 1.006 & 1.016 & 1.013 & 1.022 & 0.986 & 0.997 & 1.016 & 1.007 \\
\hline auto ARMA-MRA (2) & & 0.988 & 1.005 & 1.036 & 1.031 & 1.011 & 0.998 & 0.999 & 1.005 & 1.007 & 1.004 & 1.010 & 0.991 & 1.004 & 1.003 & 1.007 \\
\hline auto ARMA-MRA (4) & \multirow{2}{*}{16} & 0.975 & 0.975 & 0.976 & 0.990 & 1.002 & 1.000 & 1.011 & 1.025 & 1.021 & 1.023 & 1.013 & 0.985 & 0.965 & 0.965 & 0.994 \\
\hline auto ARMA-MRA (2) & & 0.992 & 0.986 & 0.989 & 1.000 & 1.016 & 1.008 & 1.015 & 1.031 & 1.023 & 1.027 & 1.018 & 1.003 & 0.997 & 1.001 & 1.008 \\
\hline auto ARMA-MRA (3) & \multirow{2}{*}{8} & 1.006 & 1.020 & 1.018 & 1.044 & 1.041 & 1.055 & 1.059 & 1.056 & 1.048 & 1.051 & 1.052 & 1.045 & 1.047 & 1.066 & 1.044 \\
\hline auto ARMA-MRA (2) & & 1.005 & 1.019 & 1.017 & 1.038 & 1.035 & 1.049 & 1.054 & 1.050 & 1.042 & 1.046 & 1.044 & 1.039 & 1.040 & 1.057 & 1.039 \\
\hline
\end{tabular}

Notes: We report the improvements in MAE based on the auto ARMA-MRA model vs. simple ARMA. The numbers presented are the ratio of MAE based on the simple ARMA model over the MAE of ARMA-MRA model. We use the MODWT with haar wavelet. In the parentheses, we show the levels of the decomposition. Window length and forecast horizon are in monthly basis. The last column reports the average improvement in MAE. 
TAble 3. Paired T-STATistics (RMSE) FOr CRUde Oil MARKets

\begin{tabular}{|c|c|c|c|c|c|c|c|c|c|c|c|c|c|c|c|}
\hline \multirow{2}{*}{ Forecasting Model } & \multirow{2}{*}{$\begin{array}{l}\text { Window } \\
\text { Length }\end{array}$} & \multicolumn{14}{|c|}{ Forecast Horizon } \\
\hline & & $\overline{1}$ & 2 & 3 & 4 & 5 & 6 & 7 & 8 & 9 & 10 & 11 & 12 & 13 & 14 \\
\hline auto ARMA-MRA (8) & \multirow{2}{*}{256} & $-2.276^{* *}$ & 0.157 & $-1.805^{*}$ & $-2.905^{* * *}$ & $-3.032 * * *$ & $-3.925 * * *$ & $-2.818^{* * *}$ & -1.297 & -0.126 & -0.546 & -0.821 & -1.293 & $-2.062 * *$ & $-1.681^{*}$ \\
\hline auto ARMA-MRA (2) & & -1.527 & 0.823 & -0.012 & $-1.655^{*}$ & $-1.831 *$ & $-2.575^{* *}$ & -1.411 & 0.705 & 1.162 & 0.911 & 0.314 & -0.143 & $-1.669 *$ & -1.125 \\
\hline auto ARMA-MRA (7) & \multirow{2}{*}{128} & $-2.486^{* *}$ & 0.084 & 0.392 & 0.463 & 0.719 & 0.539 & -0.255 & 0.341 & 0.141 & 0.409 & 1.033 & $1.731^{*}$ & 0.623 & 0.885 \\
\hline auto ARMA-MRA (2) & & -1.257 & 1.037 & 0.500 & 0.608 & 0.712 & 0.203 & -0.659 & 0.870 & 1.242 & 1.277 & $1.97 * *$ & $2.163 * *$ & 0.402 & 1.025 \\
\hline auto ARMA-MRA (6) & \multirow{2}{*}{64} & $-1.805^{*}$ & 1.596 & $1.723^{*}$ & 0.550 & 0.255 & -0.204 & -0.920 & $1.682 *$ & 1.464 & $2.382 * *$ & $1.787^{*}$ & 0.174 & 0.686 & 0.894 \\
\hline auto ARMA-MRA (2) & & -0.755 & $1.78^{*}$ & $1.889 *$ & 0.727 & 0.612 & 0.033 & -1.346 & 1.037 & 1.102 & 1.302 & 1.382 & -0.618 & -0.386 & -0.054 \\
\hline auto ARMA-MRA (5) & \multirow{2}{*}{32} & -1.107 & 0.922 & $1.722 *$ & $2.284 * *$ & $1.882^{*}$ & 0.518 & 0.713 & 0.703 & 1.551 & 1.471 & 1.209 & -0.796 & 0.400 & 0.919 \\
\hline auto ARMA-MRA (2) & & -0.161 & 1.176 & $1.969 * *$ & $2.741 * * *$ & $1.758^{*}$ & 0.165 & 0.479 & 0.604 & 1.014 & 0.758 & 1.064 & -0.874 & 0.974 & 0.838 \\
\hline auto ARMA-MRA (4) & \multirow{2}{*}{16} & 0.042 & 0.891 & 1.008 & 0.949 & 1.280 & 1.065 & 1.116 & 1.089 & 0.992 & 1.058 & 0.984 & 0.914 & 0.875 & 0.876 \\
\hline auto ARMA-MRA (2) & & 0.649 & 1.007 & 1.146 & 1.024 & 1.380 & 1.113 & 1.183 & 1.141 & 1.042 & 1.090 & 1.034 & 0.986 & 0.947 & 0.961 \\
\hline auto ARMA-MRA (3) & \multirow{2}{*}{8} & 1.217 & 1.143 & 1.132 & 1.276 & 1.176 & 1.106 & 1.108 & 1.079 & 1.043 & 1.029 & 1.025 & 1.026 & 1.017 & 1.022 \\
\hline auto ARMA-MRA (2) & & 1.254 & 1.173 & 1.151 & 1.283 & 1.178 & 1.110 & 1.114 & 1.084 & 1.045 & 1.032 & 1.025 & 1.027 & 1.018 & 1.023 \\
\hline
\end{tabular}

Notes: We report the Paired t-statistics of the difference in mean between the squared forecast errors of the auto ARMA-MRA model vs. simple ARMA. We use the MODWT with haar wavelet. In the parentheses, we show the levels of the decomposition. Window length and forecast horizon are in monthly basis. The last column reports the average improvement in rMSE. *,**,*** denotes statistical significance at $10 \%, 5 \%$ and $1 \%$, respectively. 
TAble 4. Paired t-Statistics (MAE) FOR CRude Oil Markets

\begin{tabular}{|c|c|c|c|c|c|c|c|c|c|c|c|c|c|c|c|}
\hline \multirow{2}{*}{ Forecasting Model } & \multirow{2}{*}{$\begin{array}{l}\text { Window } \\
\text { Length }\end{array}$} & \multicolumn{14}{|c|}{ Forecast Horizon } \\
\hline & & 1 & 2 & 3 & 4 & 5 & 6 & 7 & 8 & 9 & 10 & 11 & 12 & 13 & 14 \\
\hline auto ARMA-MRA (8) & \multirow{2}{*}{256} & $-2.079 * *$ & -1.163 & $-2.093 * *$ & $-2.589 * *$ & $-2.336^{* *}$ & $-3.692 * * *$ & -1.609 & -0.814 & 1.023 & -0.101 & 0.268 & -0.697 & -1.247 & -1.452 \\
\hline auto ARMA-MRA (2) & & -1.599 & -0.507 & -0.688 & -1.386 & -1.232 & -2.361 & -0.943 & 0.202 & $1.819 *$ & 1.263 & 1.111 & -0.064 & -0.691 & -1.209 \\
\hline auto ARMA-MRA (7) & \multirow{2}{*}{128} & $-2.483^{* *}$ & -0.684 & -0.223 & 0.114 & 0.137 & 0.633 & -0.440 & 0.876 & 0.563 & -0.078 & 0.583 & 0.898 & -0.066 & 0.578 \\
\hline auto ARMA-MRA (2) & & -1.593 & 0.043 & 0.693 & 0.999 & 0.840 & 0.742 & -0.237 & 1.464 & 1.184 & 0.498 & 1.486 & 1.426 & -0.195 & 1.208 \\
\hline auto ARMA-MRA (6) & \multirow{2}{*}{64} & $-1.857^{*}$ & 0.388 & 0.906 & -0.178 & -0.357 & -0.337 & -1.596 & 1.453 & 1.276 & $2.636 * * *$ & $2.634 * * *$ & 0.264 & 0.440 & 1.111 \\
\hline auto ARMA-MRA (2) & & -0.909 & 0.160 & 1.265 & 0.591 & 0.374 & 0.226 & $-1.865^{*}$ & 0.705 & 0.975 & 1.499 & $2.63 * * *$ & -0.559 & -0.014 & -0.402 \\
\hline auto ARMA-MRA (5) & \multirow{2}{*}{32} & -1.451 & -0.095 & $1.684 *$ & $2.159 * *$ & 0.924 & -0.444 & 0.139 & 0.441 & 1.135 & 1.075 & $1.727^{*}$ & -0.998 & -0.213 & 0.940 \\
\hline auto ARMA-MRA (2) & & -0.590 & 0.379 & $2.442 * *$ & $2.562 * *$ & 0.964 & -0.215 & -0.142 & 0.534 & 0.703 & 0.410 & 1.263 & -1.097 & 0.397 & 0.322 \\
\hline auto ARMA-MRA (4) & \multirow{2}{*}{16} & -1.241 & -1.234 & -1.050 & -0.418 & 0.083 & 0.013 & 0.348 & 0.720 & 0.535 & 0.546 & 0.290 & -0.338 & -0.742 & -0.699 \\
\hline auto ARMA-MRA (2) & & -0.496 & -0.849 & -0.590 & 0.014 & 0.770 & 0.358 & 0.616 & 1.188 & 0.804 & 0.845 & 0.550 & 0.093 & -0.089 & 0.022 \\
\hline auto ARMA-MRA (3) & \multirow[b]{2}{*}{8} & 0.451 & 1.283 & 0.959 & $1.998 * *$ & 1.627 & $1.951^{*}$ & $1.848^{*}$ & 1.584 & 1.225 & 1.171 & 1.164 & 0.935 & 0.923 & 1.208 \\
\hline auto ARMA-MRA (2) & & 0.462 & 1.439 & 1.108 & $2.087 * *$ & 1.641 & $2.035^{* *}$ & $1.973 * *$ & $1.655^{*}$ & 1.282 & 1.252 & 1.175 & 0.957 & 0.937 & 1.246 \\
\hline
\end{tabular}

Notes: We report the Paired t-statistics of the difference in mean between the absolute errors of the auto ARMA-MRA model vs. simple ARMA. We use the MODWT with haar wavelet. In the parentheses, we show the levels of the decomposition. Window length and forecast horizon are in monthly basis. The last column reports the average improvement in MAE. *, **, *** denotes statistical significance at 10\%, 5\% and $1 \%$, respectively. 


\section{FIGURE 1. RMSE (A) AND MAE (B) FOR THREE LEVELS OF DECOMPOSITION}

(a)

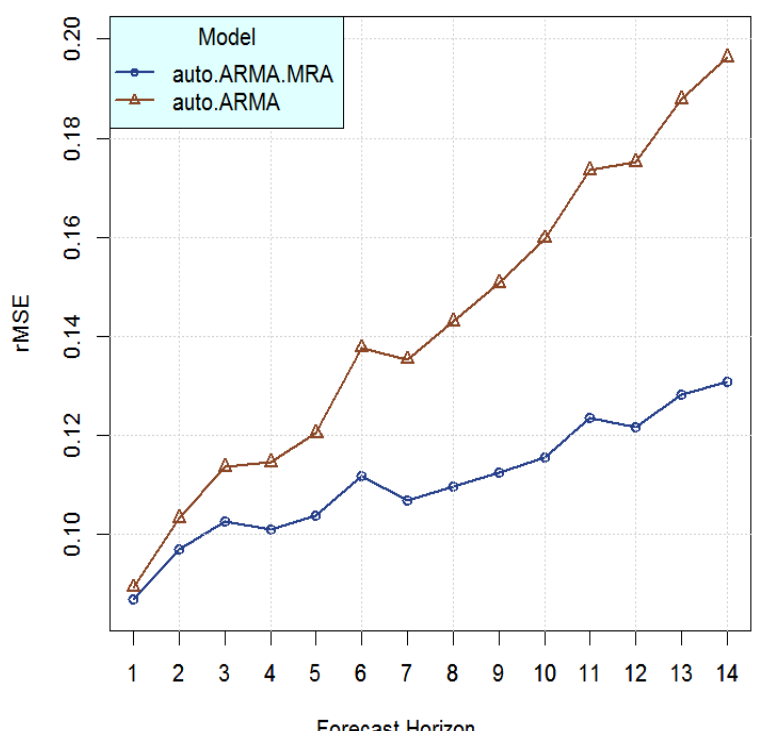

(b)

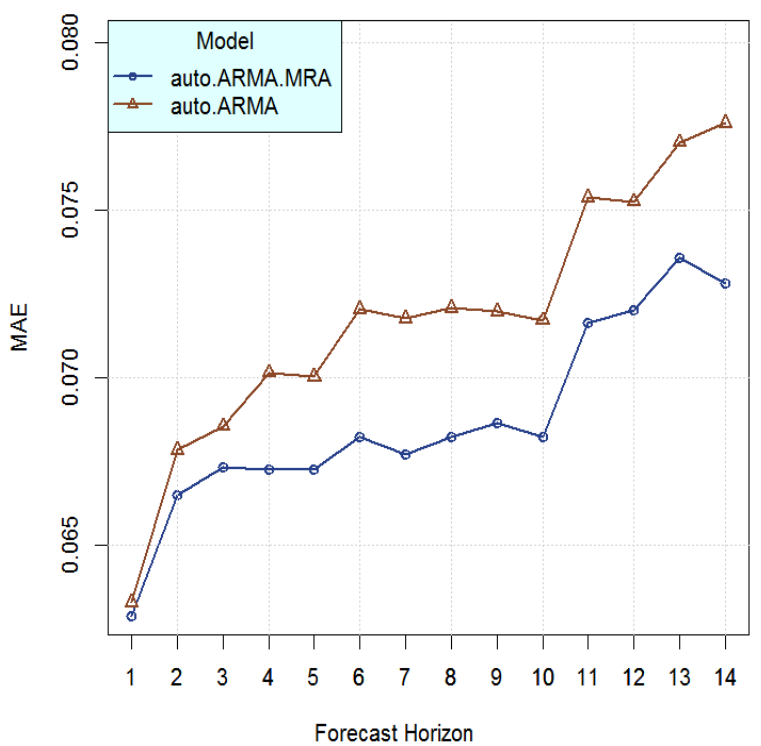

Notes: This figure compares the forecast errors rMSE (panel a) and MAE (panel b) of auto ARMA-MRA model with 3 levels of decomposition vs. the ones from simple auto ARMA model as the benchmark. The results are obtained by applying rolling window estimation. 


\section{FIGURE 2. RMSE (A) AND MAE (B) FOR FOUR LEVELS OF DECOMPOSITION}

(a)

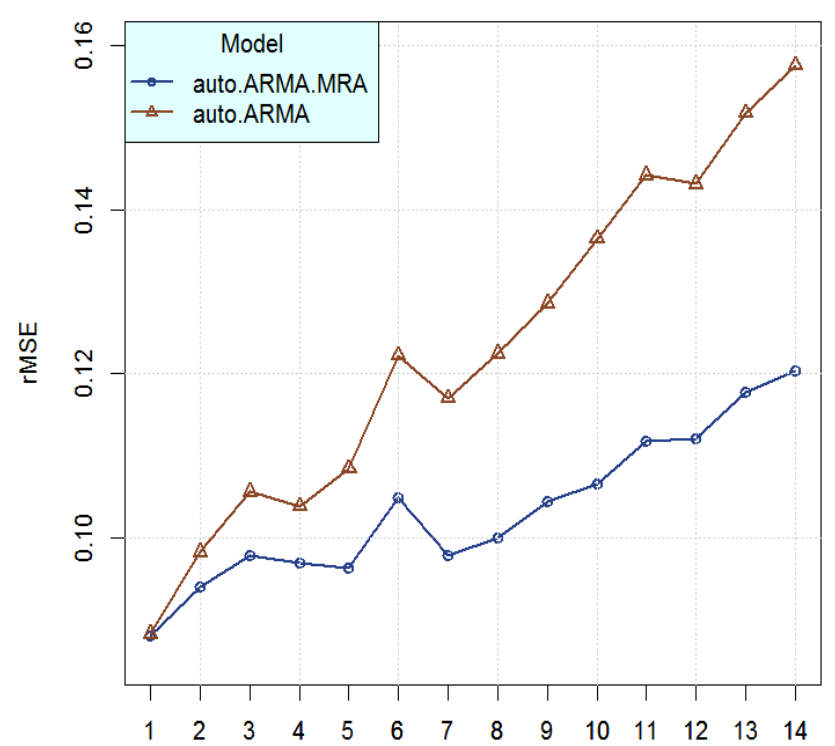

Forecast Horizon (b)

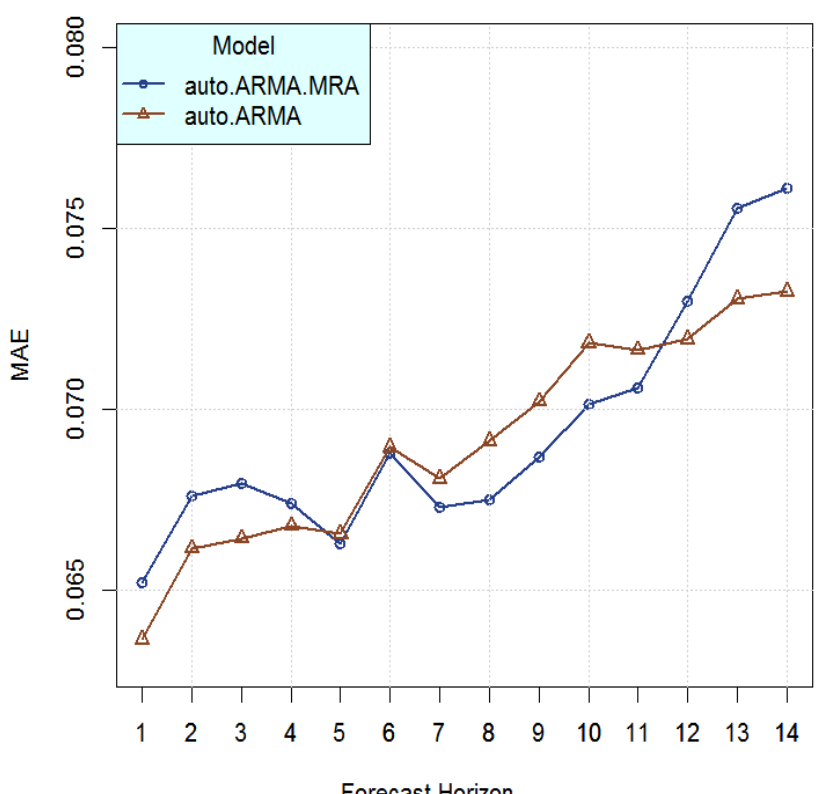

Forecast Horizon

Notes: This figure compares the forecast errors rMSE (panel a) and MAE (panel b) of auto ARMA-MRA model with 4 levels of decomposition vs. the ones from simple auto ARMA model as the benchmark. The results are obtained by applying rolling window estimation. 


\section{FigURE 3. COMPARATIVE RMSE SCORES}

rMSE for auto ARMA-MRA with 2 levels of decomposition

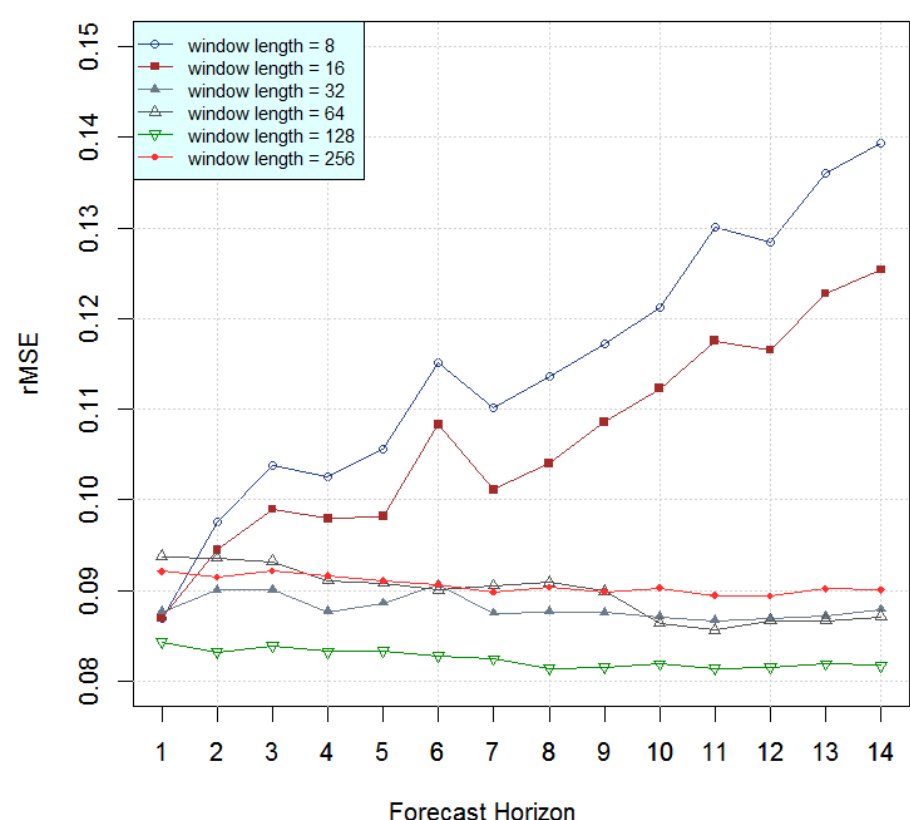

Notes: We present forecast errors (rMSE) from auto ARMA-MRA model with 2 levels of decomposition. Several training sample sizes are used and the results are obtained via rolling window estimation. Window length and forecast horizon are on a monthly basis. 